\title{
Preface
}

\section{Fat Grafting for Aesthetic Facial Surgery}

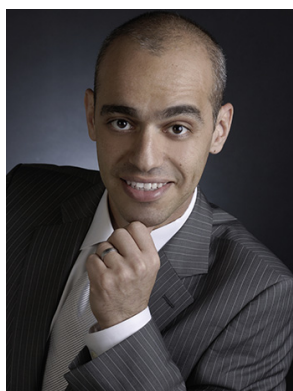

Shahrokh C. Bagheri, DMD, MD, FACS, FICD

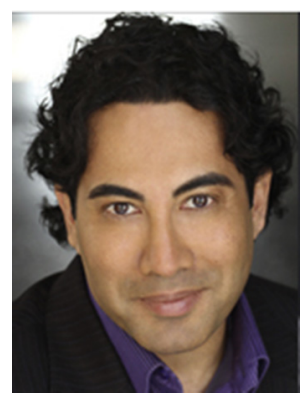

Husain Ali Khan, DMD, MD, FACS

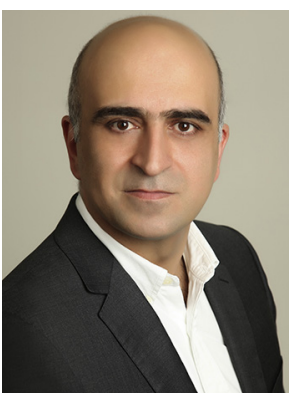

Behnam Bohluli, DMD, MS

Editors

Over the last few years, fat grafting has evolved significantly and is becoming a growing trend. Historic concepts of fat cells of simply being temporary fillers are changing as it is being discovered that fat cells are able to maintain their vitality throughout the surgical procedure and survive at the recipient site.

This paradigm shift has affected many aspects of fat-graft procedures. Even the terminology "fat injection," which implies a spiritless and empty technique, is now being referred to as a "fat transfer" or "fat graft," which emphasizes the viability of these cells.

Instruments and techniques are considerably modified in order to conservatively harvest, process, and transfer viable fat parcels to the recipient site. Moreover, many efforts are recently being made to enhance the longevity and the regenerative potentials of donor fat. Research into stem cells and platelet-derived growth factors is producing results that are opening a new era in this field of oral and maxillofacial surgery.

It is not surprising that with these advances, current indications for fat grafting (eg, facial augmentation and facial rejuvenations) are producing more predictable and have higher-quality results. In addition, these techniques are newly being used adjunctively in face lifts, blepharoplasty, revision rhinoplasty, and orthognathic surgery and are substituting some of these current aesthetic procedures as they may be less complex.

This issue of Atlas of the Oral and Maxillofacial Surgery Clinics of North America specifically covers an update on all above-mentioned areas of fat grafting, and a multidisciplinary team of authors and experts that are mostly among the world-known aesthetic surgeons may be a paramount feature of this issue. We hope this new exceptional situation will help to reveal different concepts and overlooked sides to fat grafting and it is hoped the readers find most of their questions and needs in these 11 comprehensive articles.

Shahrokh C. Bagheri, DMD, MD, FACS, FICD Georgia Oral \& Facial Reconstructive Surgery Northside Hospital

2795 Peachtree Road Northeast Suite 2008, Atlanta, GA 30305, USA

Husain Ali Khan, DMD, MD, FACS Aesthetics International USA

Facial Cosmetic Surgery 11975 Morris Road, Suite 220 Alpharetta, GA 30005, USA

Behnam Bohluli, DMD, MS 124 Edward Street

Toronto, Ontario M5G 1G6, Canada

E-mail addresses: sbagher@hotmail.com (S.C. Bagheri) husainakmd@yahoo.com (H.A. Khan) bbohluli@yahoo.com (B. Bohluli) 